\title{
REVIEW
}

\section{REVIEW OF SUCCINATE DEHYDROGENASE-DEFICIENT \\ RENAL CELL CARCINOMA WITH FOCUS ON CLINICAL AND PATHOBIOLOGICAL ASPECTS}

\author{
Naoto Kuroda $^{1}$, Kenji Yorita ${ }^{1}$, Makoto Nagasaki ${ }^{2}$, Yuji Harada ${ }^{3}$, Chisato Ohe H, $^{4,5}$ \\ Jera Jeruc ${ }^{6}$, Maria Rosaria Raspollini $^{7}$, Michal Michal $^{8}$, Ondrej Hes ${ }^{8}$, Mahul B. Amin ${ }^{4}$
}

\author{
${ }^{1}$ Department of Diagnostic Pathology, Kochi Red Cross Hospital, Kochi, Japan \\ ${ }^{2}$ Department of Clinical Laboratory, the National Hospital Organization Hamada Medical Center, Hamada, Japan \\ ${ }^{3}$ Department of Surgical Pathology, Shimane University School of Medicine, Izumo, Japan \\ ${ }^{4}$ Department of Pathology and Laboratory Medicine, Cedars-Sinai Medical Center, Los Angeles, CA, USA \\ ${ }^{5}$ Department of Diagnostic Pathology, Kansai Medical University, Hirakata Hospital, Osaka, Japan \\ ${ }^{6}$ Faculty of Medicine, Institute of Pathology, University of Ljubljana, Ljubljana, Slovenia \\ ${ }^{7}$ Department of Histopathology and Molecular Diagnostics, University Hospital Careggi, Florence, Italy \\ ${ }^{8}$ Department of Pathology, Charles University in Prague, Faculty of Medicine in Plzen, Czech Republic
}

\begin{abstract}
Succinate dehydrogenase (SDH)-deficient renal cell carcinoma (RCC) was first identified in 2004 and has been integrated into the 2016 WHO classification of RCC. Succinate dehydrogenase (SDH) is an enzyme complex composed of four protein subunits (SDHA, SDHB, SDHC and SDHD). The tumor which presents this enzyme mutation accounts for 0.05 to $0.2 \%$ of all renal carcinomas. Multiple tumors may occur in approximately $30 \%$ of affected patients. SDHB-deficient RCC is the most frequent, and the tumor histologically consists of cuboidal cells with eosinophilic cytoplasm, vacuolization, flocculent intracytoplasmic inclusion and indistinct cell borders. Ultrastructurally, the tumor contains abundant mitochondria. Immunohistochemically, tumor cells are positive for SDHA, but negative for SDHB in SDHB-, SDHC- and SDHD-deficient RCCs. However, SDHA-deficient RCC shows negativity for both SDHA and SDHB. In molecular genetic analyses, a germline mutation in the $S D H B, S D H C$ or $S D H D$ gene (in keeping with most patients having germline mutations in an $S D H$ gene) has been identified in patients with or without a family history of renal tumors, paraganglioma/ pheochromocytoma or gastrointestinal stromal tumor. While most tumors are low grade, some tumors may behave in an aggressive fashion, particularly if they are high nuclear grade, and have coagulative necrosis or sarcomatoid differentiation.
\end{abstract}

Key words: succinate dehydrogenase, renal cell carcinoma, review.

\section{Introduction}

Succinate dehydrogenase (SDH)-deficient renal cell carcinoma (RCC) was identified by Vanharata et al. in 2004 and been accepted by the 2016 WHO organization of renal tumors as a unique subtype of RCC. In
2013, it was integrated into the ISUP (International Society of Urological Pathology) Vancouver Classification published as a provisional entity $[1,2,3]$. Since its first description, some recent large studies dealing with this tumor have been reported $[4,5,6,7]$. The 
tumor entity morphologically and genetically seems to show a wider spectrum than has been hitherto recognized $[6,7,8,9,10,11]$. In this article, we review the clinicopathological aspects of this tumor.

\section{Epidemiology and clinical features}

The frequency of SDH-deficient RCC is estimated as between 0.05 to $0.2 \%$ of all renal carcinomas [7]. Among SDH-deficient RCCs, SDHB-mutated RCC is the most frequent $[1,2,3,4,5,6,7,8,10]$, followed by SDHC and SDHD-deficient RCC $[5,7,9]$. Very recently, one case with SDHA-deficient RCC has been described [11]. The lifetime risk of renal tumor in patients with the SDHB gene mutation has been estimated as $14 \%$ [12]. The age of patients ranges from 22 to 72 years, with a mean age of 40 years in Williamson's series and from 14 to 76 years with a mean age of 37 years in Gill's series $[6,7]$. There is a slight male predominance $[6,7]$. The association with paraganglioma/pheochromocytoma or type 2 gastrointestinal stromal tumor has been reported $[1$, 2, 3, 4, 5, 6]. SDH-deficient gastrointestinal stromal tumors and paragangliomas are also known to be associated with the syndromic, non-hereditary Carney triad of paraganglioma, pulmonary chondroma and SDH-deficient gastrointestinal stromal tumor. Due to the lifelong $14 \%$ risk of developing a renal neoplasm, genetic testing in the appropriate clinical context should be considered for patients with this constellation of tumors.

\section{Pathological findings}

\section{Macroscopic findings}

The size of the tumor ranges from $20 \mathrm{~mm}$ to 200 $\mathrm{mm}$ in Williamson's series and from 7 to $90 \mathrm{~mm}$ with a mean of $51 \mathrm{~mm}$ in Gill's series $[6,7]$. Gross- ly, the tumors are usually well circumscribed and the cut surface shows tan to red, tan to brown, or red to brown color $[6,7]$. Hemorrhage or partial cystic change may sometimes be observed $[6,7]$. Bilateral tumors have been reported in approximately $30 \%$ of patients $[6,7]$.

\section{Microscopic findings \\ SDHB-deficient renal cell carcinoma}

In this subtype, various patterns overlapping with other known histological subtypes including chromophobe RCC, clear cell RCC, papillary RCC, sarcomatoid RCC, unclassified RCC and renal oncocytoma have been described $[1,2,3,4,5,6,7,8,10]$. In the early reports, the morphology of the tumors was generally never illustrated [4]. Among them, oncocytic carcinoma with a unique morphology was most frequently observed $[3,4,5,6,7]$. We now recognize this as a unique tumor type with the following characteristic features: 1) the tumor is composed of nested, tubular and solid architecture with variable cysts; 2) the tumor cells are cuboidal with eosinophilic cytoplasm containing typical cytoplasmic vacuoles or flocculent inclusions which when prominent impart a bubbly appearance; 3) the nuclei are homogeneous, have smooth nuclear contours, evenly distributed chromatin and a neuroendocrine appearance with inconspicuous nucleoli [3, 4, 5, 6, 7] (Fig. 1A, B); 4) entrapped tubules or glomeruli are observed at the peripheral edges of the tumor $[6,7]$. Mast cells often infiltrate the intratumoral stroma $[6,7]$.

\section{SDHC- and SDHD-deficient renal cell carcinomas}

These tumors generally show morphology comparable with clear cell RCC [5, 9]. In one SDHC-deficient case, papillary RCC has also been described [9].
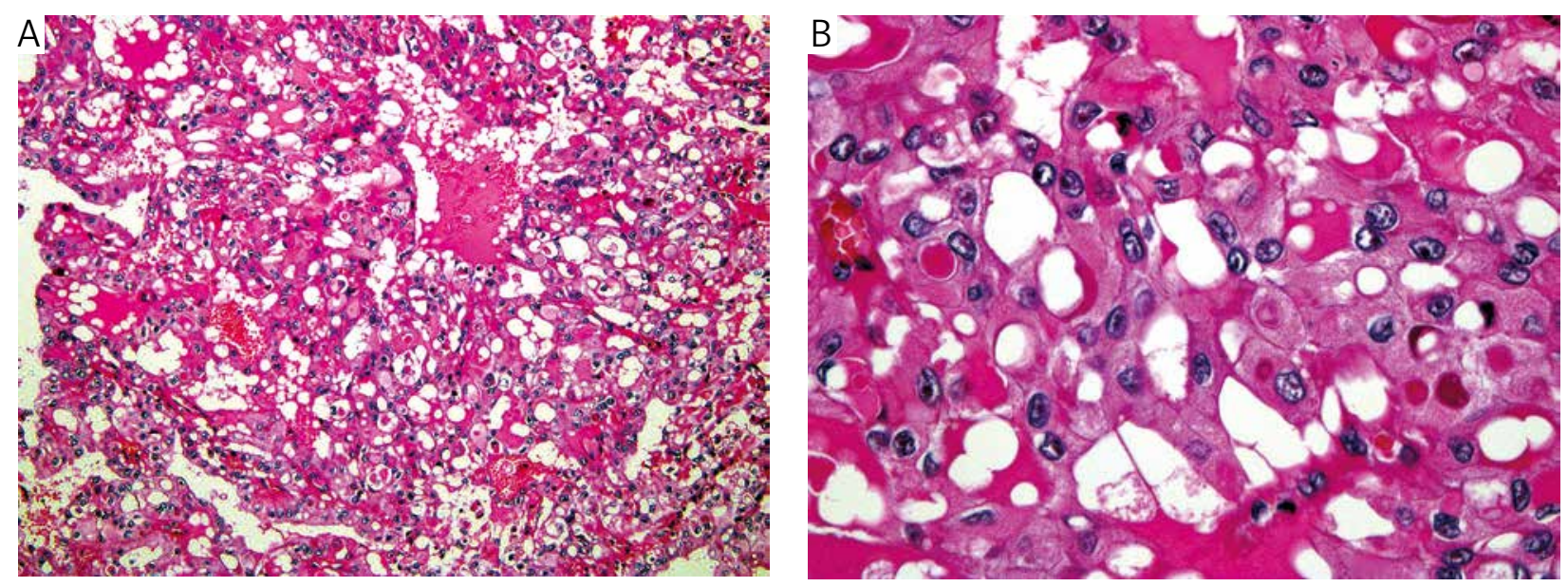

Fig. 1. Microscopic findings. A) The tumor consists of eosinophilic to oncocytic cells with multiple cytoplasmic vacuoles and an indistinct cell border. B) At higher magnification, some cytoplasmic inclusions are observed 


\section{SDHA-deficient renal cell carcinoma}

One case of SDHA-deficient RCC showed unclassified RCC characteristics of both papillary RCC and collecting duct carcinoma [11].

\section{Immunohistochemical findings}

SDH-deficient RCCs are generally immunoreactive for PAX8 and Ksp-cadherin, but negative for c-kit, RCC Ma, p63 and CA9 [3, 4, 6, 7]. The immunoreactivity for epithelial markers is negative or focal. Immunohistochemical loss of SDHB is a diagnostic requirement. In SDHB-, SDHC- and SDHD-deficient RCCs, tumor cells are negative for SDHB (Fig. 2), but positive for SDHA [3, 4, 6, 7, 13]. In contrast, tumor cells in SDHA-deficient RCC show negativity for both SDHA and SDHB [11]. Neuroendocrine markers are negative.

\section{Ultrastructural findings}

In SDHB-deficient RCC, the cytoplasm of tumorous cells contains abundant mitochondria and cytoplasmic inclusions correspond to abnormal mitochondria with degenerating cristae and ground substance [13].

\section{Molecular genetic findings}

SDH-deficient RCC is strongly hereditary with the vast majority demonstrating germline mutations in one of the SDH related genes. A germline mutation in SDHB, SDHC or SDHD has been identified $[1,2,3,4,5,6,7,9,12,13\}$; SDHB mutations are most common followed by SDHC. SDHB-deficient RCCs seem to harbor mutations that alter arginine codons, and loss of the second allele is frequent [1, 6]. In SDHC-deficient RCC, tumor cells showed loss of heterozygosity (LOH) for intragenic and flanking markers of the SDHC gene locus [9].

\section{Differential diagnosis}

The following renal tumors should be taken into consideration in the differential diagnostic process: chromophobe RCC, clear cell RCC, hybrid oncocytic/chromophobe tumor (HOCT), renal oncocytoma, renal oncocytosis, acquired cystic disease (ACD)-associated RCC, Birt-Hogg-Dubé (BHD) syndrome-associated RCC, hereditary leiomyomatosis RCC syndrome (HLRCC), and PTEN hamartoma syndrome. In chromophobe RCC, the tumor generally consists of pale and eosinophilic cells with distinct cell borders, perinuclear haloes, nuclear irregularities and occasional binucleated cells [14]. Tumor cells generally demonstrate diffuse positivity for cytokeratin 7, CD117 and Ksp-cadherin [15, 16]. Clear cell

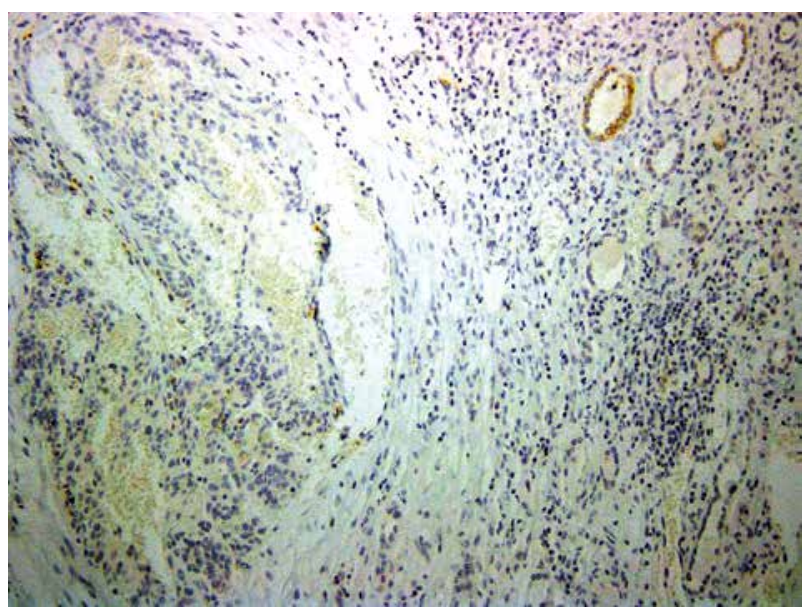

Fig. 2. Immunohistochemical findings of SDHB protein. The tumor cells are completely negative for SDHB. At contrast, normal tubules are positive

RCC generally consists of clear cells with a varying growth pattern including alveolar, solid, acinar and cystic pattern and shows diffuse membranous positivity for CA9 and vimentin [15]. The cytoplasm may sometimes be prominently eosinophilic and with the nested architecture is an important mimic of SDH-deficient RCC. The SDHB immunohistochemistry may be problematic to interpret and may be weak in clear cell carcinoma due to the abundant clear cytoplasm; this should not be interpreted as benign negative. HOCT shows hybrid morphology of both chromophobe RCC and renal oncocytoma, namely uniform-sized tumor cells with eosinophilic cytoplasm, round nuclei and perinuclear haloes. In renal oncocytoma, a nesting growth pattern is often observed in the background of edematous or hyalinized stroma $[17,18]$. Some patients with renal oncocytosis may have a history of chronic renal failure or dialysis [19]. In BHD syndrome-related RCCs, HOCT is the most frequent. The clinical association with cutaneous (fibrofolliculoma, trichodiscoma or acrochordon) and pulmonary (cyst and pneumothorax) lesions is very important. The germline mutation of the FLCN gene is very important for the definite diagnosis of BHD syndrome [20]. In HLRCC, tumor cells usually correspond to papillary RCC, type 2 and have eosinophilic prominent nucleoli resembling cytomegalovirus inclusion. Recently, the spectrum of architectural patterns has been expanded: papillary, tubulopapillary, tubular, cribriform, solid and cystic. Most patients have also cutaneous and/or uterine leiomyomas clinically [21]. However, it was reported in recent papers that synchronous or metachronous occurrence of RCC and leiomyoma is relatively rare. In PTEN hamartoma syndrome, patients have trichilemmoma, thyroid cancer, uterine corpus cancer or breast cancer clinically [22]. Additionally, tumor cells show immunohistochemical negativity for PTEN. 


\section{Therapy and clinical management}

An immediate surgical intervention should be recommended [23]. Nephron-sparing surgery should be generally selected when a solid tumor is present and is at the early stage. Active surveillance only is not recommended [24]. In advanced disease, FDG-PET examination is recommended [5]. In cases with metastatic disease, molecular targeted therapy for vascular endothelial growth factor (VEGF), mammalian target of rapamycin (mTOR), and tyrosine kinase (TK) has been previously administered $[11,25]$. We are not aware of a report where the patient was treated with ablative therapy. Some authors have proposed that if the patients exceed the age 40 to 45 and have no family history of renal tumor, the molecular analyses of SDHB may be unnecessary [8]. However, in patients under age 45 the possible diagnosis of SDH-deficient RCC should be considered even in the absence of family history [5]. Clinico-pathologic correlation is key and management in a multidisciplinary setting is optimal.

\section{Prognosis}

It is well known that a subset of SDH-deficient RCC has potential to behave aggressively $[4,5,7$, $11,23,26]$. However, tumors with low grade nuclear morphology behave in an indolent fashion after the complete resection [4]. Tumor with high grade nuclear features, coagulative necrosis or sarcomatoid dedifferentiation may behave in an aggressive fashion, and the observation of any of these features needs to be documented in the pathology report [7]. Metastases to the liver, bone, brain, lung and lymph nodes have been documented $[4,5,6,7,11,25]$.

\section{Future perspectives}

In the screening for the detection of SDH-deficient RCC, the immunohistochemistry of both SDHA and SDHB proteins is available $[4,5,7,27]$. However, pathologists need to pay attention to the immunohistochemical interpretation of these proteins, because clear cell RCC tends to show more false negatives for SDHB protein than other histological types [28]. In SDHA-deficient RCC, whether or not germline mutation of the SDHA gene exists remains unknown [11]. Accordingly, a more consistent number of cases and the germline mutation analysis of the SDHA gene are necessary. In order to clarify the relationship between SDHC- or SDHD-deficient RCCs and histological type or clinical behavior, further examination in a large scale study will be needed.

The authors declare no conflict of interest.

\section{References}

1. Vanharanta S, Buchta M, McWhinney SR, et al. Early-onset renal cell carcinoma as a novel extraparaganglial component of SDHB-associated heritable paraganglioma. Am J Hum Genet 2004; 74: 153-159.

2. Gill A, Amin MB, Smith S, Trpkov K. Succinate dehydrogenase-deficient renal carcinoma. WHO Classification of Tumors of the Kidney, Bladder and Male Genital tract. Moch H, Humphrey P, Reuter VE (eds). IARC Press, Lyon.

3. Srigley JR, Delahunt B, Eble JN, et al. The International Society of Urological Pathology (ISUP) Vancouver Classification of renal neoplasia. Am J Surg Pathol 2013; 37: 1469-1489.

4. Gill AJ, Pachter NS, Chou A, et al. Renal tumors associated with germline SDHB mutation show distinct morphology. Am J Surg Pathol 2011; 35: 1578-1585.

5. Ricketts CJ, Shuch B, Vocke CD, et al. Succinate dehydrogenase kidney cancer (SDH-RCC): An aggressive example of the Warburg effect in cancer. J Urol 2012; 188: 2063-2071.

6. Williamson SR, Eble JN, Amin MB, et al. Succinate dehydrogenase-deficient renal cell carcinoma: detailed characterization of 11 tumors defining a unique subtype of renal cell carcinoma. Mod Pathol 2015; 28: 80-94.

7. Gill AJ, Hes O, Papathomas T, et al. Succinate dehydrogenase (SDH)-deficient renal carcinoma: a morphologically distinct entity. A clinicopathologic series of 36 tumors from 27 patients. Am J Surg Pathol 2014: 38: 1588-1602.

8. Eng C. SDHB - a gene for all tumors? J Natl Cancer Inst 2008; 100: 1193-1195.

9. Malinoc A, Sulivan M, Wiech T, et al. Bialleic inactivation of the SDHC gene in renal carcinoma associated with paraganglioma syndrome type 3. Endocrine-Related Cancer 2012; 19 : 283-290.

10. Haas NB, Nathanson KL. Hereditary kidney cancer syndrome. Avd Chr Kid Dis 2014; 21: 81-90.

11. Yakirevich E, Ali SM, Mega A, et al. A novel SDHA-deficient renal cell carcinoma revealed by comparative genomic profiling. Am J Surg Pathol 2015; 39: 858-863.

12. Ricketts CJ, Forman JR, Rattenberry E, et al. Tumor risks and genotype-phenotype analysis in 358 patients with germline mutations in SDHB and SDHD. Hum Mutat 2010; 31: 4151.

13. Housley SL, Lindsay RS, Young B, et al. Renal carcinoma with giant mitochondria associated with germ-line mutation and somatic loss of the succinate dehydrogenase B. Histopathology 2010; 56: 401-408.

14. Amin MB, Paner GP, Alvarado-Cabrero I, et al. Chromophobe renal cell carcinoma: histomorphologic characteristics and evaluation of conventional pathologic prognostic parameters in 145 cases. Am J Surg Pathol 2008; 32: 1822-1834.

15. Kuroda N, Tanaka A, Ohe C, et al. Recent advances of immunohistochemistry for diagnosis of renal tumors. Pathol Int 2013; 63: 381-390.

16. Kuehn A, Paner GP, Skinnider BF, et al. Expression analysis of kidney-specific cadherin in a wide spectrum of traditional and newly recognized renal epithelial neoplasms: diagnostic and histogenitic implications. Am J Surg Pathol 2007; 31: 15281533.

17. Petersson F, Gatalica Z, Grossmann P, et al. Sporadic hybrid oncocytic/chromophobe tumor of the kidney: a clinicopathologic, histomorphologic, immunohistochemical, ultrastructural, and molecular genetic study of 14 cases. Virchows Arch 2010; 456: 355-365.

18. Hes O, Petersson F, Kuroda N, et al. Renal hybrid oncocytic/ chromophobe tumors - a review. Histol Histopathol 2013; 28: 1257-1264.

19. Kuroda N, Tanaka A, Ohe C, et al. Review of renal oncocytosis (multiple oncocytic lesions) with focus on clinical and pathobiological aspects. Histol Histopathol 2012; 27: 1407-1412. 
20. Kuroda N, Furuya M, Nagashima Y, et al. Review of renal tumors associated Birt-Hogg-Dubé syndrome with focus on clinical and pathobiological aspects. Pol J Pathol 2014; 65: 93-99.

21. Merino MJ, Torres-Cabala C, Pinto P, et al. The morphologic spectrum of kidney tumors in hereditary leiomyomatosis and renal cell carcinoma (HLRCC) syndrome. Am J Surg Pathol 2007; 31: 1578-1585.

22. Shuch B, Ricketts CJ, Vocke CD, et al. Germline PTEN mutation Cowden syndrome: An underappreciated form of hereditary kidney cancer. J Urol 2013; 190: 1990-1998.

23. Linehan WM, Ricketts CJ. The metabolic basis of kidney cancer. Semin Cancer Biol 2013; 23: 46-55.

24. Bratslavsky G, Linehan WM. Long-term management of bilateral, multifocal, recurrence renal carcinoma. Nat Rev Urol 2010; 7: 267-275.

25. Paik JY, Toon CW, Benn DE, et al. Renal carcinoma associated with succinate dehydrogenase $\mathrm{B}$ mutation: $\mathrm{A}$ new and unique subtype of renal carcinoma. J Clin Oncol 2014; 32: e10-e14.

26. Shuch B, Linehan WM, Srinlvasan R. Aerobic glycolysis: A novel target in kidney cancer. Expert Rev Anticancer Ther 2013; 13: 711-719.

27. Gill AJ, Pachter NS, Clarkson A, et al. Renal tumors and hereditary pheochromocytoma-paraganglioma syndrome type 4 . N Engl J Med 2011; 364: 885-886.

28. Cornejo KM, Lu M, Yang P, et al. Succinate dehydrogenase B: a new prognostic biomarker in clear cell renal cell carcinoma. Hum Pathol 2015; 46: 820-826.

\section{Address for correspondence}

Naoto Kuroda, MD

Department of Pathology

Kochi Red Cross Hospital

Shin-honmachi 2-13-51, Kochi City

Kochi 780-8562, Japan

tel. +81-88-822-1201

fax $+81-88-822-1056$

e-mail:kurochankochi@yahoo.co.jp 